\title{
DETECTION OF MULTI-TeV GAMMA RAYS FROM MARKARIAN 501 DURING AN UNFORESEEN FLARING STATE IN 1997 WITH THE TIBET AIR SHOWER ARRAY
}

\author{
M. Amenomori ${ }^{1}$, S. Ayabe ${ }^{2}$, P.Y. Cao $^{3}$, Danzengluobu ${ }^{4}$, L.K. Ding ${ }^{5}$, Z.Y. Feng ${ }^{6}$, Y. Fu ${ }^{3}$,

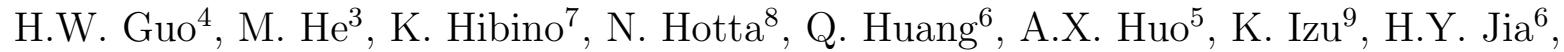 \\ F. Kajino ${ }^{10}$, K. Kasahara ${ }^{11}$, Y. Katayose ${ }^{9}$ Labaciren $^{4}$, J.Y. Li ${ }^{3}$, H. Lu ${ }^{5}$, S.L. Lu ${ }^{5}$,

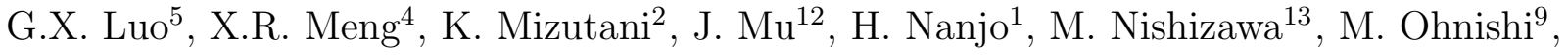 \\ I. Ohta ${ }^{8}$, T. Ouchi ${ }^{7}$, J.R. Ren ${ }^{5}$, T. Saito ${ }^{14}$, M. Sakata ${ }^{10}$, T. Sasaki ${ }^{10}$, Z.Z. Shi ${ }^{5}$, \\ M. Shibata ${ }^{15}$, A. Shiomi ${ }^{9}$, T. Shirai ${ }^{7}$, H. Sugimoto ${ }^{16}$, K. Taira ${ }^{16}$, Y.H. Tan ${ }^{5}$, N. Tateyama ${ }^{7}$, \\ S. Torii ${ }^{7}$, T. Utsugi ${ }^{2}$, C.R. Wang $^{3}$, H. Wang ${ }^{5}$, X.W. Xu ${ }^{5}$, Y. Yamamoto ${ }^{10}$, G.C. Yu ${ }^{6}$, \\ A.F. Yuan ${ }^{4}$, T. Yuda ${ }^{9}$, C.S. Zhang ${ }^{5}$, H.M. Zhang ${ }^{5}$, J.L. Zhang ${ }^{5}$, N.J. Zhang ${ }^{3}$, X.Y. Zhang ${ }^{3}$, \\ Zhaxiciren $^{4}$, Zhaxisangzhu ${ }^{4}$, and W.D. Zhou ${ }^{12}$ (The Tibet AS $\gamma$ Collaboration)
}

\begin{abstract}
In 1997, the BL Lac Object Mrk 501 entered a very active phase and was the brightest source in the sky at $\mathrm{TeV}$ energies, showing strong and frequent flaring.
\end{abstract}

\footnotetext{
${ }^{1}$ Department of Physics, Hirosaki University, Hirosaki 036-8561, Japan

2 Department of Physics, Saitama University, Urawa 338-8570, Japan

${ }^{3}$ Department of Physics, Shangdong University, Jinan 250100, China

${ }^{4}$ Department of Mathematics and Physics, Tibet University, Lhasa 850000, China

${ }^{5}$ Institute of High Energy Physics, Academia Sinica, Beijing 100039, China

${ }^{6}$ Department of Physics, South West Jiaotong University, Chengdu 610031, China

7 Faculty of Engineering, Kanagawa University, Yokohama 221-8686, Japan

${ }^{8}$ Faculty of Education, Utsunomiya University, Utsunomiya 321-8505, Japan

${ }^{9}$ Institute for Cosmic Ray Research, University of Tokyo, Tanashi 188-8502, Japan

10 Department of Physics, Konan University, Kobe 658-8501, Japan

11 Faculty of Systems Engineering, Shibaura Institute of Technology, Omiya 330-8570, Japan

12 Department of Physics, Yunnan University, Kunming 650091, China

13 National Center for Science Information Systems, Tokyo 112-8640, Japan

14 Tokyo Metropolitan College of Aeronautical Engineering, Tokyo 116-0003, Japan

15 Faculty of Engineering, Yokohama National University, Yokohama 240-0067, Japan

16 Shonan Institute of Technology, Fujisawa 251-8511, Japan
} 
Using the data obtained with a high density air shower array that has been operating successfully at Yangbajing in Tibet since 1996, we searched for $\gamma$-ray signals from this source during the period from February through August in 1997. Our observation detected multi-TeV $\gamma$-ray signals at the $3.7 \sigma$ level during this period. The most rapid increase of the excess counts was observed between April 7 and June 16 and the statistical significance of the excess counts in this period was $4.7 \sigma$. Among several observations of flaring $\mathrm{TeV} \gamma$-rays from Mrk 501 in 1997, this is the only observation using a conventional air shower array. We present the energy spectrum of $\gamma$-rays which will be worthy to compare with those obtained by imaging atmospheric Cerenkov telescopes.

Subject headings: gamma rays : observations - BL Lacertae objects : individual (Markarian 501)

\section{INTRODUCTION}

Mrk 501 and Mrk 421 have been well detected as extra-galactic TeV $\gamma$-ray sources by Whipple and subsequent ground-based Cerenkov detectors (Ong 1998). They are the so-called BL Lac objects, which are radio-loud AGNs (Active Galactic Nuclei) whose relativistic jets are aligned along our line of sight. Flux variability on various scales is a common feature of BL Lac objects as already seen in Mrk 501 and Mrk 421, and spectral variations of $\gamma$-rays coming from these sources are considered to be a very powerful tool for understanding the physics of BL Lac objects. When Mrk 501 was first detected by the Whipple Collaboration in 1995 (Quinn et al. 1996), it showed rather low fluxes at a level significantly below the Crab flux. In March of 1997, however, this source went into a state of remarkably flaring activity and its high state lasted for almost half a year with highly variable and strong $\gamma$-ray emission. The maximum flux reached roughly 10 times that of the Crab. During this period, several groups (Protheroe et al. 1997) observed strong $\gamma$-ray emission from this source with imaging atmospheric Cerenkov detectors. Independent measurements of the $\gamma$-ray spectrum seem to show a gradual softening towards higher energy, while the systematic uncertainties in the flux estimates remain too large to reach a common understanding. The energy spectrum and its shape are very important quantities for clarifying the mechanism of $\gamma$-ray production or particle acceleration at the source, and eventually to lead to the actual measurement of the intergalactic infrared or far-infrared background field (Stecker \& de Jager 1993). Hence, confirmation of the detection of $\gamma$-rays with a different technique will be strongly required. 
The Tibet air shower array, operating since 1990, is located at Yangbajing (4300 m above sea level) in Tibet (Amenomori et al. 1992). This array has a capability of detecting $\gamma$-rays in the $\mathrm{TeV}$ energy region with high efficiency and good angular resolution. Using this array, we have succeeded in detecting the Crab at the $5.5 \sigma$ level (Amenomori et al. 1999). In this paper we present the observation of multi-TeV $\gamma$-ray flares from Mrk 501 in 1997. The result obtained with well established air shower technique is important for comparing with those by imaging atmospheric Cerenkov telescopes.

\section{EXPERIMENT}

The Tibet air shower array consists of two overlapping arrays (Tibet-II and HD) as described elsewhere (Yuda 1996). The Tibet-II array comprises 185 scintillation detectors (BICRON 408A) of $0.5 \mathrm{~m}^{2}$ each placed on a $15 \mathrm{~m}$ square grid with an enclosed area of 36,900 $\mathrm{m}^{2}$, and the HD (high density) array is operating inside the Tibet-II array to detect cosmic ray showers with energies lower than $10 \mathrm{TeV}$. This HD array consists of 109 scintillation detectors (some of detectors are commonly used in both arrays), placed on a $7.5 \mathrm{~m}$ square grid covering an area of $5,175 \mathrm{~m}^{2}$. The detector arrangement of the Tibet air shower array is schematically shown in Fig. 1. Every detector, except those placed with a $30 \mathrm{~m}$ spacing on the outskirts of the inner detector matrix of the Tibet-II array, is equipped with a fast timing (FT) phototube (HPK H1161) and is thus referred to as an "FT-detector". A lead plate of $5 \mathrm{~mm}$ thickness is placed on the top of each detector to improve the fast timing data by converting $\gamma$-rays in the showers to electron pairs. This lead converter typically increases the shower size by a factor of about 2 and improves the angular resolution by about $30 \%$ (Amenomori et al. 1990).

All the TDCs (time-to-digital converters) and ADCs (analog-to-digital converters) are regularly monitored by using a calibration module in the FASTBUS system at every 20 minutes. The length of each signal cable is also monitored by measuring a mismatched-reflection pulse from each detector. The data-taking system has been operating under any 4-fold coincidence in the FT-detectors, resulting in that the trigger rate of the events is about $200 \mathrm{~Hz}$ for the Tibet-II while being about $115 \mathrm{~Hz}$ for the HD array.

The observation presented here was made by using the data taken between 1997 February and 1997 August. The event selection was done by imposing the following three conditions to the recorded data : 1) Each of any four FT detectors should record a signal of more than 1.25 particles ; 2) among the four detectors recording the highest particles, two or more should be within each detector area of the Tibet II and HD arrays denoted by the dotted and solid lines, respectively, in Fig.1 ; and 3) the zenith angle of the incident 
direction should be less than $45^{\circ}$. After data processing and quality cuts, the total number of events selected were $5.5 \times 10^{8}$ for the HD array and $1.0 \times 10^{9}$ for the Tibet-II array, respectively, with the effective running time of 155.3 days.

\section{ARRAY PERFORMANCE}

Since the background cosmic rays are isotropic and $\gamma$-rays from a source are apparently centered on the source direction, a bin size for collecting on-source data should be determined based on the array's angular resolution so as to optimize the signal to noise ratio. In order to achieve a good resolution, a study of core-finding techniques and shower-front curvature corrections has been done (Amenomori et al. 1990). The angular accuracy of the Tibet array can be checked thoroughly by observing the shadow that the Moon casts in the cosmic rays (Amenomori et al. 1993). The Tibet II and HD arrays have a capability of observing the Moon's shadow with good statistics. The mode energies of primary protons to be detected are about $3 \mathrm{TeV}$ and about $8 \mathrm{TeV}$ for the Tibet HD and II arrays, respectively. Hence the angular resolution of each array can be independently examined in respective energy regions. The statistical significance of the Moon's shadow observed with both arrays becomes about $10 \sigma$ or more for half a year observation. From this observation, we estimated the angular resolution of both arrays to be better than $0.9^{\circ}$ for all events. We have also found that the angular resolution scales with $\sum \rho$, where $\sum \rho$ stands for the sum of the number of shower particles per $\mathrm{m}^{2}$ detected in each counter. The resolution increases with increasing $\sum \rho$ as $0.8^{\circ} \times\left(\left(\geq \sum \rho\right) / 20\right)^{-0.3}\left(15<\sum \rho<300\right)$.

The Moon's shadow by the events with $\sum \rho=15-50$ was found at the position shifted from the Moon center to the west by $0.32^{\circ}\left( \pm 0.10^{\circ}\right)$. The primary cosmic rays casting the Moon's shadow are almost protons and the mean energy of protons capable of generating these events at Yangbajing is estimated to be about $4.7 \mathrm{TeV}$ by the simulation. On the other hand, a proton of energy E impinging at normal angle on the Earth is deflected by the geomagnetic field and its deflection angle is calculated as $\Delta \theta E \simeq 1.6^{\circ} \mathrm{TeV}$. So, the observed shift of the Moon's shadow is consistent with that expected from the effect of the geomagnetic field. A more elaborate study of the Moon's shadow using a Monte Carlo technique shows almost same results as those by the experiment (Suga et al. 1999). Thus, the results obtained by assigning primary energies to the observed events can be directly checked by observing the Moon's shadow.

The pointing of the array is inferred from the position of the Moon's shadow by high energy cosmic rays $(>20 \mathrm{TeV})$ which are negligibly affected by the geomagnetic field. This estimation can also be done by examining the deviations of the Moon's shadow in the 
north-south direction, since the effect of the geomagnetic field acts only in the east-west direction. It is then found to be smaller than $0.1^{\circ}$ for both arrays.

Figure 2 shows the cumulative deficit counts of the events coming from the direction of the Moon as a function of MJD, obtained with the HD array. The data set used are between February 1997 and August 1997, just corresponding to the observation period of Mrk 501. A linearly increasing of the deficit events may be a sure guarantee against the long-term stability of the array operation. Th meridian zenith angle of the Moon at Yangbajing changes between $12^{\circ}$ and $50^{\circ}$ every 27.3 days. Naturally the most efficient observations are done when the Moon comes in sight around the smallest zenith angle of about $12^{\circ}$ every 27.3 days. This effect will be found as a tier-like structure on the deficit curve, as seen in Fig. 2.

\section{RESULTS AND DISCUSSIONS}

A circular window was used to search for signals and then its size was determined based on the angular resolution estimated by the experiment. The window size is chosen to optimize the significance of signals defined by $N_{s} / N_{B}^{1 / 2}$, where $N_{S}$ is the number of signals and $N_{B}$ the number of background events, and to contain more than $50 \%$ of the signals from a source. The radii of search windows used for the events with $\sum \rho>15,50$ and 100 were $0.9^{\circ}, 0.8^{\circ}$ and $0.5^{\circ}$, respectively. The signals were searched for by counting the number of events coming from the on-source window. The background was estimated by averaging over events falling in the ten off-source windows adjacent to the source, but without overlapping each other. The source window traverses a path in local coordinates expressed by the zenith angle and azimuth angle through every day. In order to reduce a strong zenith angle dependence of the background, these off-source windows were taken in the azimuth angle directions with the same zenith angle, except two windows adjacent to the on-source window.

Figure 3 shows the cumulative excess counts for all events as a function of MJD and background, obtained with the HD array. No excess counts were observed until the middle of March 1997. However, excess events rapidly increased in the period from April through June and then it became slightly dull. The operation of the array was stopped on August 25 of 1997 to calibrate the operation system. As discussed in $§ 3$, one should first note that the observed excess counts are by no means due to some artificial noise or unstable operation of the system. The statistical significance of the excess counts reached a $3.7 \sigma$ level during this period. The excess counts very rapidly increased during the period from April 7 through June 16 and the statistical significance of the excess counts was a $4.7 \sigma$. These observed 
features are almost consistent with other observations by atmospheric Cerenkov telescopes (Protheroe et al. 1997).

Shown in Fig. 4 is the contour map of the excess event densities around Mrk 501 for the events with $\sum \rho>15$ observed between April 7 and June 16 in 1997. This map was obtained using the same method as done for the Moon and Sun shadows (Amenomori et al. 1993). Mrk 501 is well observed in the right direction by our air shower array.

Figure 5 shows the distribution of the opening angles relative to the Mrk 501 direction for all events with $\sum \rho>15$ in the HD array. The excess in the small opening angle region (less than $0.5^{\circ}$ ) could be attributed to $\gamma$-rays from Mrk 501. The simulation result done for $\gamma$-ray events coming from Mrk 501 can well reproduce the experiment as shown in Fig. 5, when we take account of the systematic pointing errors estimated in $\S 3$. For the observation period from 1997 February to 1997 August, the statistical significances of the excess events with $\sum \rho>15,30$ and 50 were $3.7 \sigma, 2.3 \sigma$ and $1.6 \sigma$, respectively.

We also searched for $\gamma$-ray emission using the entire Tibet-II array, but no excess was found in this period and upper limits on the excess number of the events at the $90 \%$ confidence level were obtained.

We estimated the $\gamma$-ray spectrum from Mrk 501 by a Monte Carlo simulation (we used a GENAS code by Kasahara \& Torii (Kasahara \& Torii 1991).), assuming a differential power-law spectrum with the form $E^{-\beta}$ and the cut-off at a certain energy, $E_{c}$, where the cut-off means that the spectral slope steepens by 1.0 at $E_{c}$. The value of $\beta$ was changed between 2.4 and 2.7 and also the effect of $E_{c}$ was examined between $7 \mathrm{TeV}$ and $30 \mathrm{TeV}$. Primary $\gamma$-rays with energies between $0.2 \mathrm{TeV}$ and $50 \mathrm{TeV}$ were thrown from the direction of Mrk 501. Observation of simulated events at Yangbajing level was done as in our experiment, estimating the collecting area, trigger efficiency and threshold energy for $\gamma$-rays generating the events at observation level. Simulated events in respective size $\left(\sum \rho\right)$ bins were then compared with those by the experiment. The energy of $\gamma$-rays was defined as the energy of the maximum flux of simulated events observed in each size bin. These steps were repeated until the observed results are well reproduced. A combination of $\beta \cong 2.6$ and $E_{c} \sim$ 20-30 TeV can reproduce the data well. We examined that the absolute flux values except the highest energy bin stay almost unchanged for above trials, but it is of course difficult to settle the spectral slope from this experiment because of very small energy range fitted here. The systematic errors on the flux arise mainly from the event selection procedure, which depends upon the array performance, and from the calculations of the collecting area and the air shower size distribution by the simulation. They are estimated to be $13 \%$ and $8 \%$, respectively (Amenomori et al. 1999). 
Shown in Figs. 6 and 7 are the energy spectra averaged in the period from February 15 to August 25 in 1997 and from February 15 to June 8 in 1997, respectively. The latter observation time corresponds to that of the Whipple Collaboration (Samuelson et al. 1998). It is seen that the results reported recently by other experiments (Samuelson et al. 1998; Hayashida et al. 1998 \& Konopelko et al. 1999) are almost compatible with ours, although these do not cover the same observation times. It should pay attention, however, that our results were obtained by the continuous observation of Mrk 501 extending February through August in 1997, while those by Cerenkov telescopes were obtained for very limited periods of moonless and cloudless nights.

Mrk 501 and Mrk 421, nearby AGNs, are at almost the same red-shift (0.033 and 0.031, respectively) and have been detected in TeV energies (Ong 1998). In particular, Mrk 501 during the strong, long-lasting 1997 flare provided a good opportunity to study the energy spectrum of $\gamma$-rays from this source in detail (Protheroe et al. 1997), suggesting a spectral feature different with that of Mrk 421 (Krennrich et al. 1999). In both sources, it is likely that a synchrotron-inverse Compton picture plays an important part (Protheroe et al. 1997). Since the attenuation mechanism of $\mathrm{TeV} \gamma$-rays by intergalactic infra-red photon field is almost the same for both sources, a difference of spectral features, if any, could be attributed to the production mechanism of $\gamma$-rays at the sources. Therefore, it is very important to continue the observation of high energy $\gamma$-rays from both sources with as small uncertainties as possible.

\section{SUMMARY}

Mrk 501 suddenly came into a very active phase from March in 1997, with several large flares and lasted for $\sim 1 / 2 \mathrm{yr}$. The maximum $\gamma$-ray flux during this period reached about 10 times as high as the Crab Nebula. Following a successful observation of steady emission of multi-TeV $\gamma$-rays from the Crab(Amenomori et al. 1999), we further detected multi-TeV $\gamma$-rays from Mrk 501 which was in a high flaring state between March 1997 and August 1997, and estimated the absolute fluxes of $\gamma$-rays around multi-TeV region, using the high resolution Tibet air shower array. The detection of a signal from this source was achieved by the improvement of the array performance, which can be directly checked by observing the Moon's shadow. Monthly observations of the Moon's shadow provide a direct check of the angular resolution, pointing accuracy, and also the stable operation of the array over a long period. Furthermore, the observation of the displacement of the Moon's shadow due the effect of the geomagnetic field provides an important check of the results obtained by assigning energies to all the events. This is the first attempt to be done in the air shower 
experiments, and it suggests that the Moon is a unique cosmic-ray anti-source capable of calibrating the array performance thoroughly. Hence, the results obtained by the Tibet experiment using a different technique will be a great help to understand the possible bias and errors involved in the Cerenkov observations.

The area of the present HD array will be extended by a factor of about five in 1999, while its effective area will be increased by a factor of about seven by the reduction of edge effects. Then, the Tibet array could cover the energy range from $3 \mathrm{TeV}$ to $\sim 100 \mathrm{TeV}$ with significantly better statistics and angular resolution at high energies. Air shower arrays are wide aperture and high duty cycle instruments, in contrast to atmospheric Cerenkov telescopes with relatively narrow fields of view and small duty cycle of $\sim 10 \%$. These features will be indispensable for understanding a time variability of emission of high energy $\gamma$-rays from point sources such as AGNs and GRBs (gamma ray bursts). The Tibet experiment, therefore, will have unique capabilities for the discovery of new, relatively bright sources and for a general survey of the overhead sky.

This work is supported in part by Grants-in-Aid for Scientific Research and also for International Science Research from the Ministry of Education, Science, Sports and Culture in Japan and for International Science Research from the Committee of the Natural Science Foundation and the Academy of Sciences in China.

\section{REFERENCES}

Amenomori, M. et al. 1990, Nucl. Instrum. Methods Phys. Res., A,288, 619

Amenomori, M. et al. 1992, Phys. Rev. Lett., 69, 2468

Amenomori, M. et al. 1993, Phys. Rev. D, 47, 2675

Amenomori, M. et al. 1999, ApJ, 525, L93

Hayashida, N. et al. 1998, ApJ, 504, L71

Kasahara, K. \& Torii, S. 1991, Comput. Phys. Commun., 64, 109

Konopelko, A.K. et al. 1999, astro-ph/9901093

Krennrich et al. 1999, ApJ, 511, 149

Ong, R.A. 1998, Phys. Rep., 305, 93 
Protheroe, R.J. et al. 1997, Proc. 25th Int. Cosmic Ray Conf. (Durban), 8, 317

Quinn, J. et al. 1996, ApJ, 456, L83

Samuelson, F.W. et al. 1998, ApJ, 501, L17

Stecker, F.W. \& de Jager, O.C. 1993, ApJ, 415, L71

Suga, Y. et al. 1999, Proc. 26th Int. Cosmic-Ray Conf. (Salt Lake City), 7, 202

Yuda, T. 1996, Proc. Int. Symp. on Extremely High Energy Cosmic Rays : Astrophysics and Future Observations, ed. M. Nagano (Tokyo : Univ. Tokyo/Inst. Cosmic-Ray Res.), 175

This preprint was prepared with the AAS IATEX macros v4.0. 


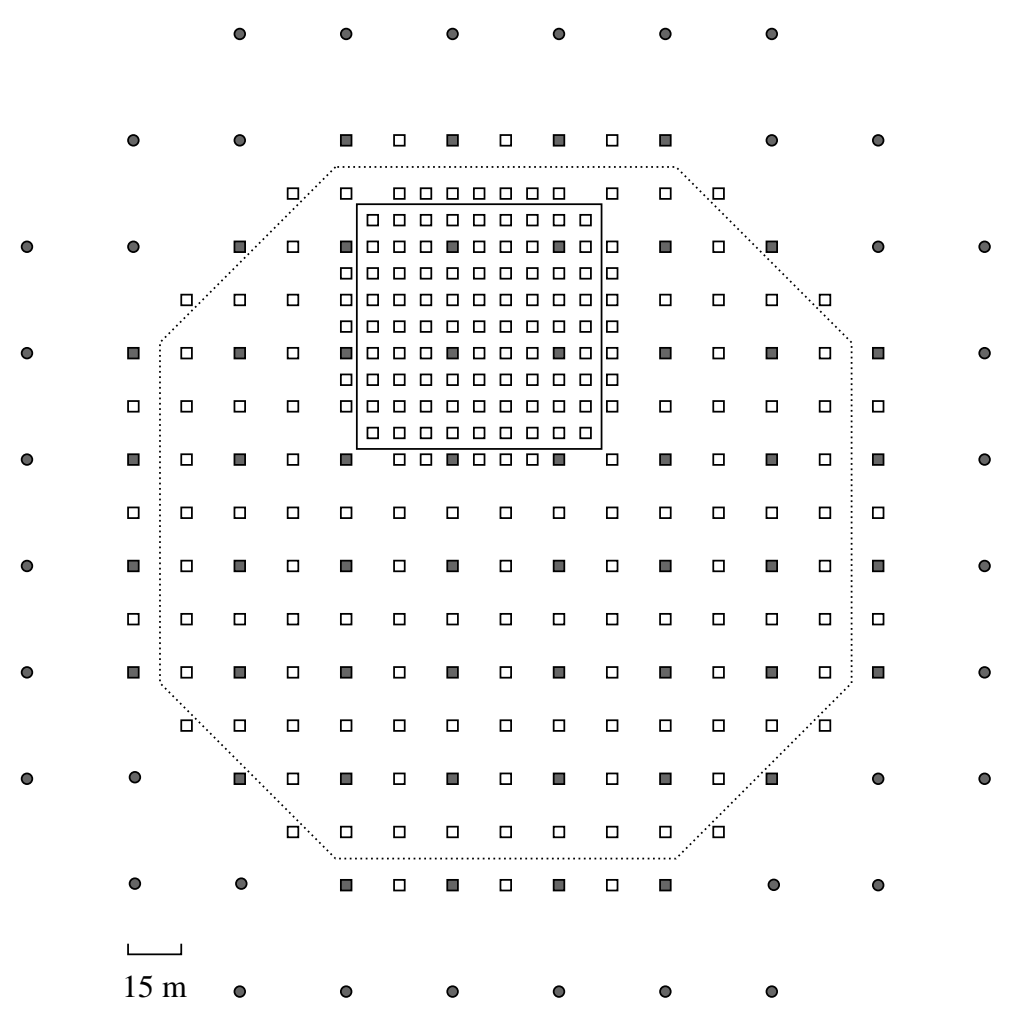

Fig. 1. - Schematical view of the Tibet-II/HD shower array operating at Yangbajing. Open and filled squares : FT-detectors ; filled circles : density detectors equipped with wide dynamic range phototube. We selected the events whose cores are within the detector matrix enclosed with the solid (HD) or dotted (Tibet-II) line. 


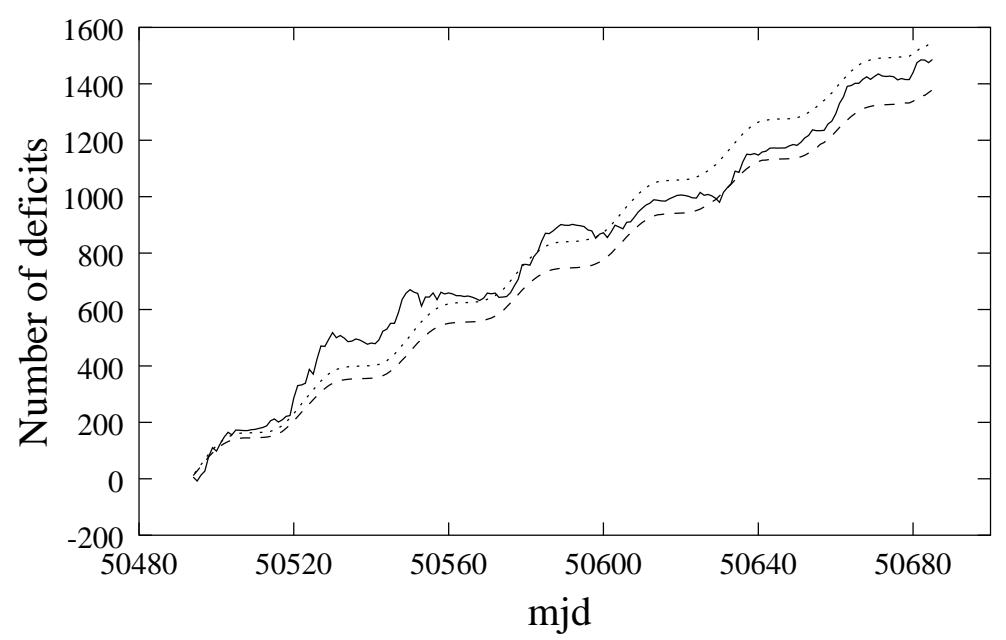

Fig. 2.- Cumulative deficit counts of events with $\sum \rho>15$ coming from the direction of the Moon as a function of MJD (solid line). The radius of search window is taken to be $0.9^{\circ}$ and its center is put on the most deficit posittion of the Moon shadow. The dotted and dashed lines denote the expected curves when the angular resolutions are assumed to be $0.8^{\circ}$ and $0.9^{\circ}$, respectively.

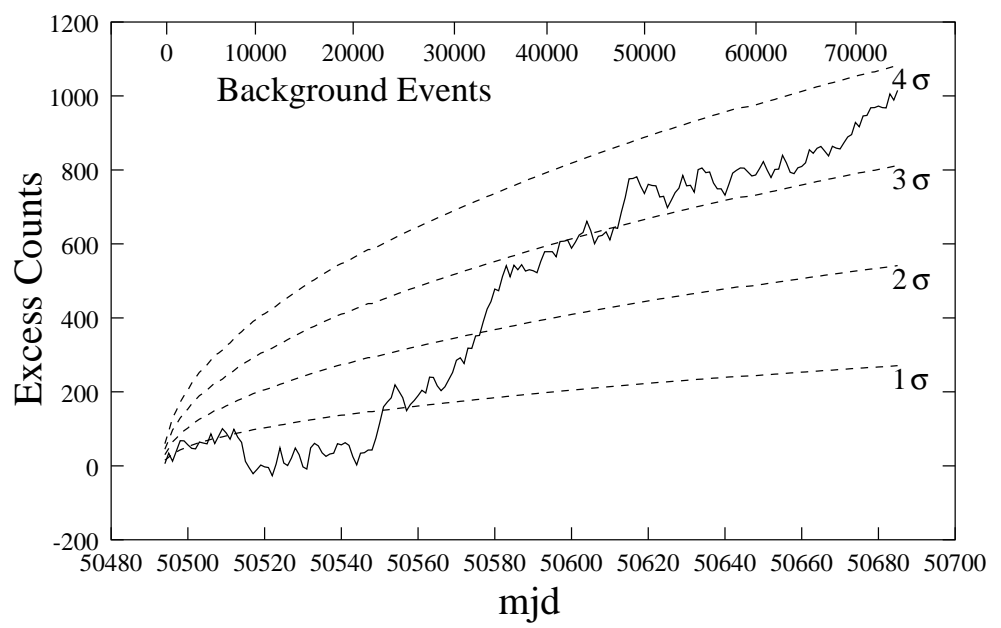

Fig. 3.- Cumulative excess of the events with $\sum \rho>15$. The dotted lines denote the excess counts at the 1, 2, 3 and $4 \sigma$ level, respectively. 


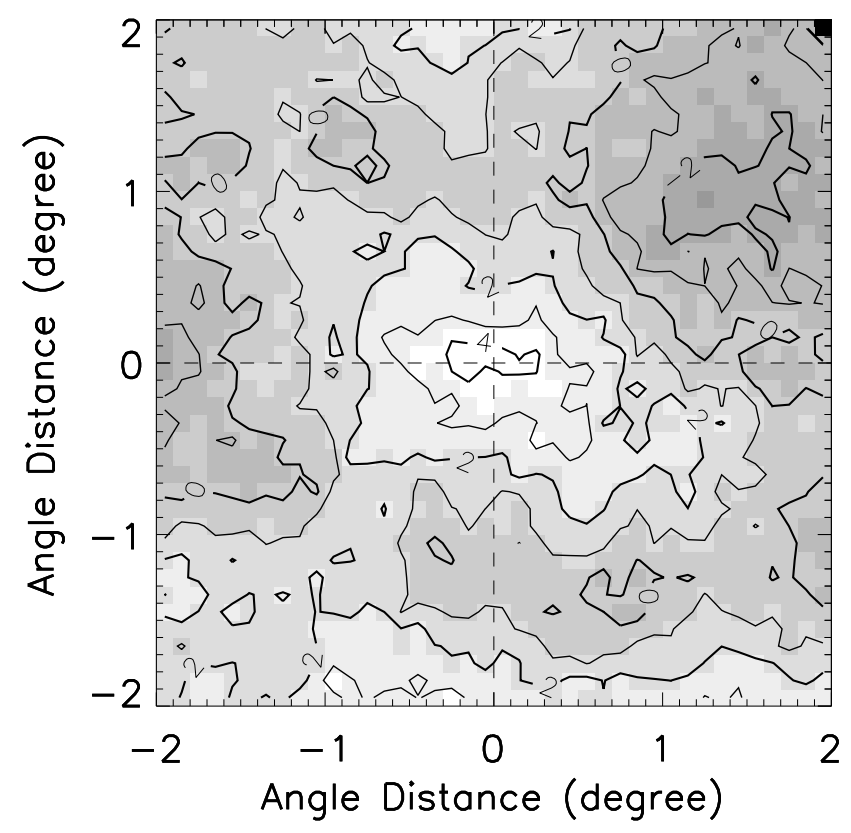

Fig. 4.- Contour map of the weights of excess event densities around Mrk 501, observed between April 7 and June 16 in 1997, in the area of $4^{\circ} \times 4^{\circ}$ centered on the direction of Mrk 501. The contour lines are drawn with a step of $1 \sigma$. Angle distance is measured from the direction of Mrk 501 along the right ascension (abscissa) and the declination (ordinate).

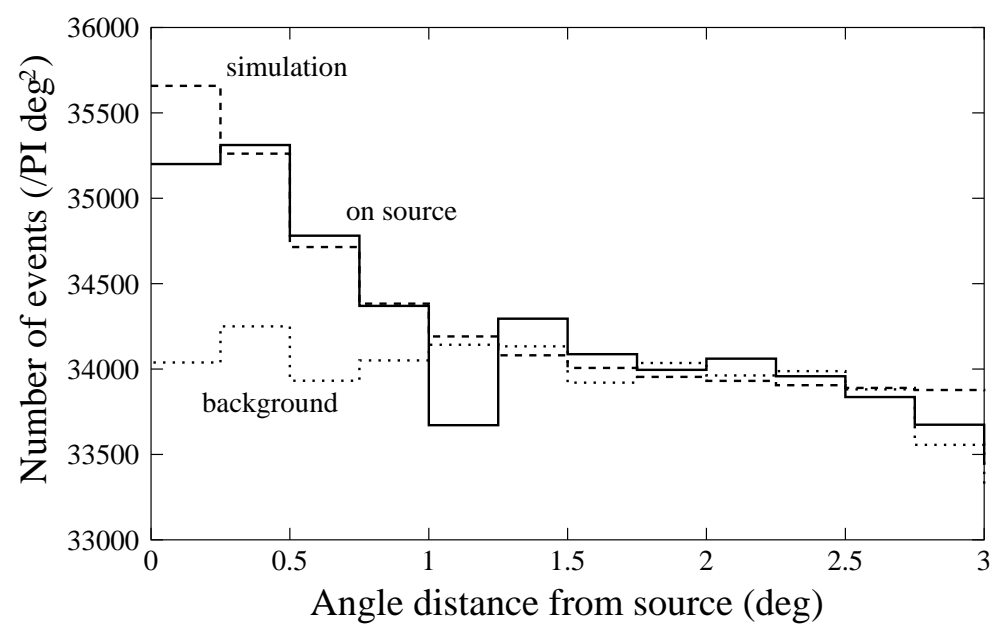

Fig. 5.- Opening angle distribution of the events with $\sum \rho>15$ coming from the directions around Mrk 510. The simulation result done for $\gamma$-ray events is shown by the dashed line. 


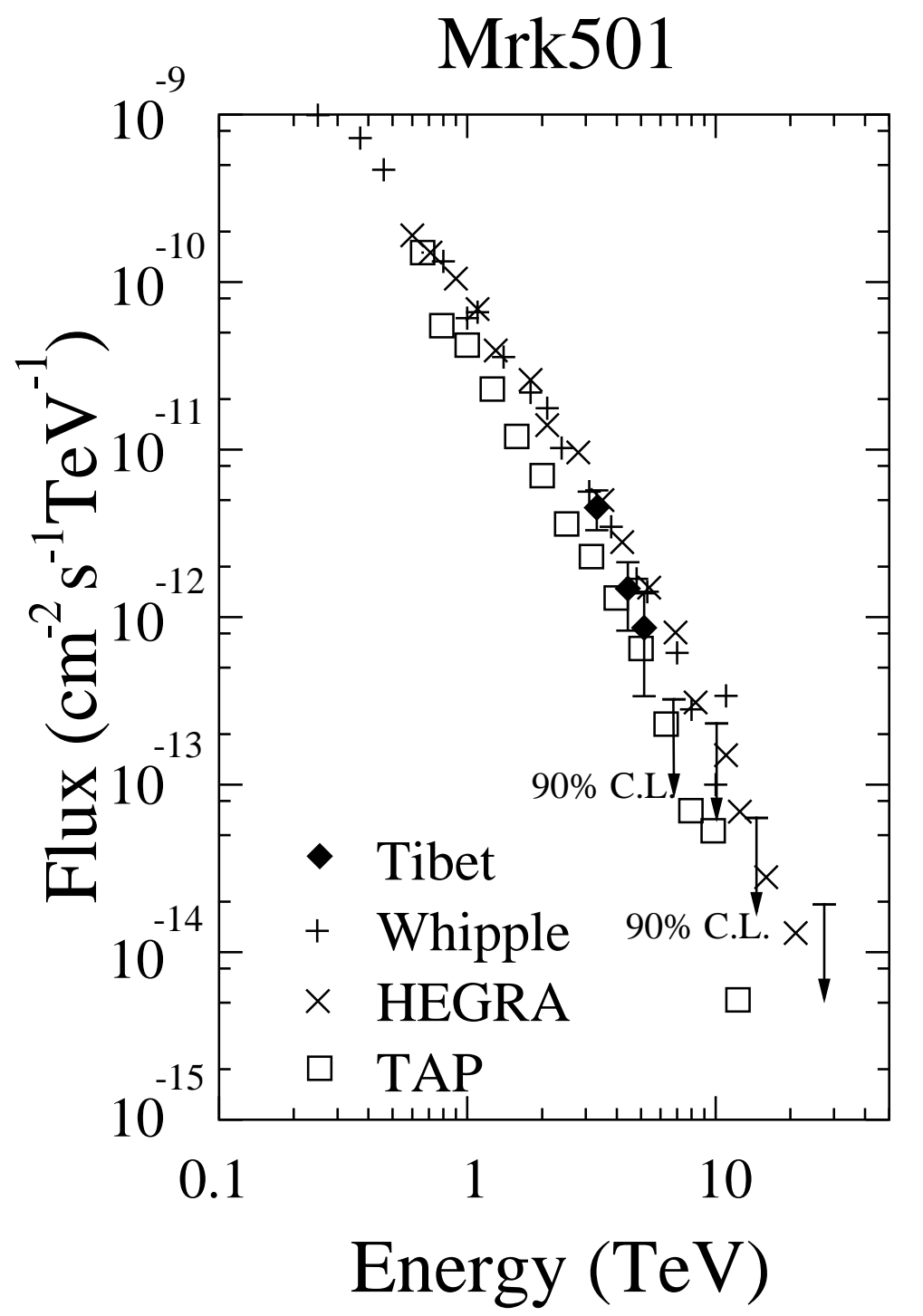

Fig. 6. - Energy spectrum of $\gamma$-rays from Mrk 501 averaged in the period from 1997 February 15 to 1997 August 25. The error bars indicate $1 \sigma$ ranges, excluding systematic errors. Upper limits at the $90 \%$ confidence level, obtained from the Tibet-II and HD arrays, are also plotted in this figure. Our data are compared with other results by Whipple (Samuelson et al. 1998 ), HEGRA (Konopelko et al. 1999) and TAP (Hayashida et al. 1998). 


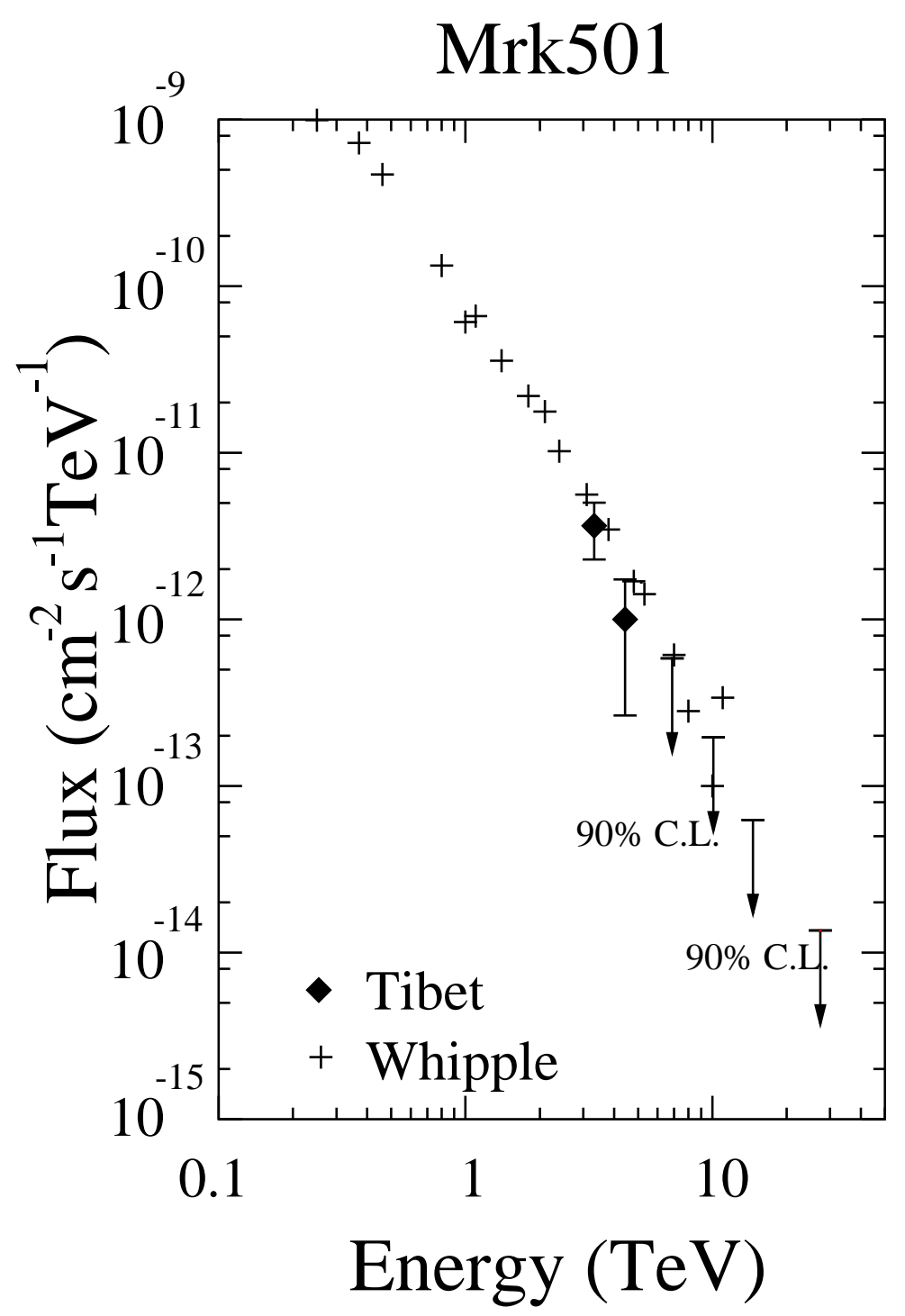

Fig. 7.-Energy spectrum of $\gamma$-rays from Mrk 501 averaged in the period from 1997 February 15 to 1997 June 9. Upper limits, obtained from the Tibet-II and HD arrays, are at the $90 \%$ confidence level. Our data are compared with the Whipple results. 